\title{
INFILTRAÇÃO DE ÁGUA NO SOLO EM ÁREAS CULTIVADAS COM CANA-DE- -AÇÚCAR SOB DIFERENTES SISTEMAS DE COLHEITA E MODELOS DE AJUSTES DE EQUAÇÕES DE INFILTRAÇÃO
}

\author{
BRUNO A. TOMASINI ${ }^{1}$, ANTONIO C. T. VITORINO², MARCOS V. GARBIATE ${ }^{3}$, \\ CRISTIANO M. A. DE SOUZA ${ }^{4}$, TEODORICO A. SOBRINHO ${ }^{5}$
}

\begin{abstract}
RESUMO: As técnicas de colheita da cultura de cana nem sempre seguem preceitos conservacionistas, fato que pode influenciar atributos físicos do solo com consequente alteração da velocidade de infiltração de água no solo. O trabalho realizado teve como objetivo avaliar o efeito de diferentes sistemas de colheita (manual e mecanizada) da cana-de-açúcar (crua e queimada), sobre as características de infiltração e verificar a adequação de modelos matemáticos para a estimativa da taxa de infiltração de água no solo. Foi utilizado um simulador de chuva portátil calibrado para aplicar uma precipitação de $60 \mathrm{~mm} \mathrm{~h}^{-1}$. Os testes de infiltração de água no solo foram realizados em três sistemas de colheita: colheita manual de cana queimada, colheita mecanizada de cana crua e colheita mecanizada de cana queimada. A qualidade do ajuste dos modelos foi avaliada por meio de regressões não lineares entre os valores estimados e os valores médios observados em cada tratamento estudado. Nos tratamentos onde foi empregada a colheita mecânica, observou-se uma redução da taxa de infiltração final em relação à área sob colheita manual de cana queimada. A equação de Horton foi a mais adequada para a estimativa da taxa de infiltração de água no solo estudado.
\end{abstract}

PALAVRAS-CHAVE: equação de horton, sistemas de colheita, infiltrômetro de aspersão.

\section{WATER INFILTRATION IN SOIL CULTIVATED WITH SUGARCANE: UNDER DIFERENT CROPPING SYSTEMS AND MODELS OF ADJUSTMENT OF INFILTRATION EQUATIONS}

\begin{abstract}
The sugarcane harvesting techniques do not always follow the precepts of soil conservation, which may influence soil physical properties with consequent change in the rate of soil water infiltration. The study aimed to evaluate the effect of different cropping systems (manual cutting and automated cutting) of sugarcanes (green and burned), on water infiltration in the soil and to determine the adequacy of mathematical models to estimate the rate of water infiltration in the soil profile. It was used a portable rainfall simulator calibrated to apply rainfall of $60 \mathrm{~mm} \mathrm{~h}^{-1}$. Tests of water infiltration in the soil were carried out in three cropping systems: manual harvesting of burnt cane, mechanical harvest of green cane and mechanical harvesting of burnt cane. The quality of the adjustment of models was evaluated by non-linear regression between the estimated values and the mean observed for each treatment. In the treatment where mechanical harvesting was applied it was observed a decreasing in the final infiltration rate compared to the area under manual harvesting of burnt cane. The equation of Horton was the most suitable to estimate the water infiltration rate in soil.
\end{abstract}

KEYWORDS: sugar cane harvest, infiltration rate, sprinkler infiltrometer.

\footnotetext{
${ }^{1}$ Eng $^{\mathrm{o}}$ Agrônomo, Universidade Federal da Grande Dourados - MS.

${ }^{2}$ Eng $^{\mathrm{O}}$ Agrônomo, Professor Associado da Faculdade de Ciências Agrárias - UFGD, Dourados - MS, Bolsista CNPq-PQ 2F, Fone: (0XX67) 3410.2411, vitorino@ufgd.edu.br.

${ }^{3}$ Aluno de Agronomia, UFGD, Dourados - MS.

${ }^{4}$ Eng ${ }^{0}$ Agrícola, Professor Adjunto, Faculdade de Ciências Agrárias - UFGD, Dourados - MS, Bolsista CNPq-PQ 2.

${ }^{5}$ Eng $^{\mathrm{O}}$ Agrônomo, Prof. Dr. Associado II, Departamento de Hidráulica e Transportes, Universidade Federal de Mato Grosso do Sul, Campo Grande - MS, Bolsista do CNPq-PQ 2.
}

Recebido pelo Conselho Editorial em: 19-2-2010

Aprovado pelo Conselho Editorial em: 9-9-2010 


\section{INTRODUÇÃO}

O Brasil é o maior produtor mundial de cana-de-açúcar e de etanol, atualmente com uma estimativa de 8 milhões de hectares de área plantada, com produção anual de 660 milhões de toneladas de açúcar e 27 bilhões de litros de etanol, de acordo com o Instituto Brasileiro de Geografia e Estatística-IBGE (2009). A necessidade mundial por fontes alternativas de energia coloca o País em posição de destaque em relação à produção de etanol, em razão das condições edafoclimáticas favoráveis ao cultivo da cana-de-açúcar, o que tem levado à expansão geográfica dessa cultura e a impactos ambientais decorrentes do seu manejo (SEVERIANO et al., 2009).

Dessa maneira, espera-se que a incorporação de novas áreas para a produção dessa cultura,seja feita respeitando-se as práticas de manejo que reduzam os impactos negativos no meio, favorecendo a sustentabilidade do sistema. No manejo da cultura da cana, as técnicas de colheita atualmente são realizadas de duas maneiras: colheita de cana queimada e colheita de cana crua, ambas podendo ser realizadas de forma manual ou mecânica. O conjunto de procedimentos envolvidos na colheita pode influenciar na produção e longevidade da cultura, nos atributos físicos, químicos e biológicos do solo, no ambiente e na saúde pública (SOUZA et al., 2006).

A prática de queima dos canaviais, amplamente utilizada no Brasil, tem como objetivo facilitar as operações de corte e manejo. Porém tal sistema de colheita traz importantes impactos ambientais, como eliminação da matéria seca da área e aumento da concentração de gás carbônico na atmosfera, contribuindo assim com o efeito estufa e com a diminuição da matéria orgânica no solo (SOUZA et al., 2005). A queima promove, também, a degradação de atributos físicos do solo por meio da redução do diâmetro médio ponderado dos agregados estáveis e pelo aumento da densidade do solo nas camadas mais superficiais, com consequente diminuição da velocidade de infiltração instantânea de água no solo (CEDDIA et al.,1999).

Por outro lado, no sistema de colheita de cana crua, ocorre a manutenção da palha sobre o solo, o que, segundo PANACHUKI et al. (2006a), é o fator mais importante no favorecimento da taxa de infiltração de água durante as chuvas. BEZERRA \& CANTALICE (2006), avaliando o efeito de diferentes coberturas do solo em área de cultivo de cana-de-açúcar sobre o escoamento superficial na erosão entressulcos, verificaram que os efeitos do dossel e do resíduo da cana-de-açúcar promoveram o aumento da rugosidade hidráulica e dos volumes de interceptação pela vegetação, reduzindo o escoamento de água, aumentando os volumes de infiltração e proporcionando menores taxas de desagregação do solo. SILVA et al. (2006) e PANACHUKI et al. (2006b) também afirmam a grande importância prática da taxa de infiltração e suas relações com o deflúvio superficial responsável pelo fenômeno da erosão. Segundo PAIXÃO et al. (2009), o conhecimento da taxa de infiltração da água no solo é fundamental para definir técnicas de conservação do solo, planejar e dimensionar sistemas de irrigação e drenagem, bem como auxiliar na composição de uma imagem mais real da retenção da água e aeração do solo.

Para descrever o processo de infiltração de água no solo, diversas equações ou modelos foram desenvolvidos, alguns a partir de considerações físicas, enquanto outros, de forma empírica. Esses modelos apresentam coeficientes que podem ser calculados a partir das equações teóricas ou serem estimados por meio de regressão a partir de dados de infiltração medidos no campo.

O presente trabalho teve por objetivo estudar o efeito de diferentes sistemas de colheita (manual e mecanizada) da cana-de-açúcar (crua e queimada), utilizados no Estado de Mato Grosso do Sul sobre a infiltração de água no solo, e verificar a adequação dos modelos matemáticos de Horton, Kostiakov-Lewis e Philip para a estimativa da taxa de infiltração de água no solo.

\section{MATERIAL E MÉTODOS}

O estudo foi realizado no município de Naviraí - MS, em solo classificado como Latossolo Vermelho distrófico (EMBRAPA, 1999) de textura média, com declividade média de 3\%, sendo os testes de campo realizados logo após a colheita da cana, no ano de 2008. O clima regional 
classificado pelo sistema internacional de Köeppen é do tipo Cwa, clima úmido e inverno seco, com precipitação média anual de $1.500 \mathrm{~mm}$ e temperatura média anual de $22{ }^{\circ} \mathrm{C}$. Alguns atributos físicos do solo estudado são apresentados na Tabela 1.

$\mathrm{Na}$ área onde se utilizou colheita mecanizada, independentemente de ser crua ou queimada, o corte da cana foi realizado por uma colhedora Modelo (A7700) com rodado de esteira, sendo a cana recolhida com trator Modelo BH180 com peso de embarque lastrado de $7.290 \mathrm{kgf}$, com rodado de 18.4-26R1 e 24.5-32R1, puxando transbordo com pneus de baixa pressão e alta flutuação.

A taxa de infiltração de água no solo foi determinada a campo, utilizando-se do simulador de chuvas portátil denominado InfiAsper, desenvolvido por ALVES SOBRINHO et al. (2002). Os testes de campo com o simulador foram realizados em três sistemas de colheita, correspondentes aos tratamentos: colheita manual de cana queimada, colheita mecanizada de cana crua e colheita mecanizada de cana queimada.

TABELA 1. Valores médios de alguns atributos físicos em um Latossolo Vermelho distroférrico nas respectivas camadas e sistemas de colheita da cana-de-açúcar. Average values of some physical properties in a Distroferric Red Latossol in the layers (cm) and in the harvest systems of sugar cane.

\begin{tabular}{|c|c|c|c|}
\hline \multirow[b]{2}{*}{ Classe textural } & \multicolumn{3}{|c|}{ Tratamentos } \\
\hline & Manual Queimada & Mecânica Crua & Mecânica Queimada \\
\hline & \multicolumn{3}{|c|}{ Textura $\left(\mathrm{g} \mathrm{kg}^{-1}\right)$} \\
\hline Argila & 155,74 & 130,49 & 155,62 \\
\hline Silte & 27,86 & 23,13 & 25,60 \\
\hline Areia & 816,01 & 846,99 & 818,78 \\
\hline Profundidade $(\mathrm{cm})$ & \multicolumn{3}{|c|}{ Macroporosidade (\%) } \\
\hline$\overline{0-5}$ & 26,11 & 15,36 & 17,46 \\
\hline $5-10$ & 26,70 & 18,86 & 12,03 \\
\hline $10-20$ & 23,54 & 16,87 & 13,28 \\
\hline Profundidade $(\mathrm{cm})$ & \multicolumn{3}{|c|}{ Microporosidade (\%) } \\
\hline $0-5$ & 22,51 & 23,43 & 22,78 \\
\hline $5-10$ & 24,47 & 24,78 & 24,32 \\
\hline $10-20$ & 26,72 & 24,98 & 24,15 \\
\hline Profundidade $(\mathrm{cm})$ & \multicolumn{3}{|c|}{ Porosidade Total $(\%)$} \\
\hline $0-5$ & 48,62 & 38,80 & 40,24 \\
\hline $5-10$ & 51,17 & 43,65 & 36,36 \\
\hline $10-20$ & 50,26 & 41,85 & 37,43 \\
\hline Profundidade $(\mathrm{cm})$ & \multicolumn{3}{|c|}{ Densidade do solo $\left(\mathrm{g} \mathrm{cm}^{-3}\right)$} \\
\hline $0-5$ & 1,52 & 1,75 & 1,73 \\
\hline $5-10$ & 1,54 & 1,61 & 1,84 \\
\hline $10-20$ & 1,59 & 1,70 & 1,80 \\
\hline
\end{tabular}

O delineamento experimental utilizado foi o de blocos casualizados, com seis repetições, totalizando 18 testes de infiltração, considerando os diferentes sistemas de condução da colheita da cana. O simulador foi calibrado para aplicar a intensidade de precipitação de $60 \mathrm{~mm} \mathrm{~h}^{-1}$, utilizando-se bicos Veejet 80.150 e pressão de $32 \mathrm{kPa}$. Antes de cada determinação no campo com o simulador de chuvas, foram coletadas amostras de solo com auxílio de um trado para determinação de umidade inicial do solo. As amostras foram retiradas nas profundidades de 0 a 0,$05 ; 0,05$ a $0,10 \mathrm{e}$ de 0,10 a $0,20 \mathrm{~m}$, com seis repetições.

A medição do escoamento superficial deu-se em intervalos sucessivos, considerando os tempos de $1 ; 2 ; 5 ; 10$ e $15 \mathrm{~min}$, com cinco repetições para cada intervalo, até que o escoamento se tornasse constante, enquanto em cada intervalo de tempo considerado foi coletado e medido o 
volume de água escoado durante $1 \mathrm{~min}$, ou seja, ao iniciar o escoamento coletava-se, durante $1 \mathrm{~min}$, o volume escoado, aguardava-se o intervalo de $1 \mathrm{~min}$ e, em seguida, coletava-se o volume novamente durante $1 \mathrm{~min}$. Este procedimento era repetido cinco vezes e, então, passava-se para o intervalo seguinte de $2 \mathrm{~min}$, coletando-se o volume escoado durante 1 min e aguardavam-se $2 \mathrm{~min}$, repetindo-se cinco vezes. O volume escoado era considerado estabilizado ou constante no tempo, quando pelo menos três sucessivos valores medidos desse volume fossem iguais.

Com base na relação entre o volume de água escoado e a área de $0,70 \mathrm{~m}^{2}$ da parcela-teste que recebia a precipitação, foi determinada a lâmina de escoamento superficial, sendo a lâmina de água infiltrada obtida pela diferença entre a lâmina de água aplicada e a lâmina de escoamento superficial, em cada intervalo de tempo. Assim, a taxa de infiltração foi obtida pela relação entre lâmina de água infiltrada e tempo de infiltração considerado.

Com os dados de infiltração obtidos nos testes a campo, foram ajustadas equações para taxa de infiltração acumulada em função do tempo, conforme modelos propostos por Kostiakov-Lewis, Horton e Philip, descritos por BRANDÃO et al. (2006). As equações para estimar a taxa de infiltração em um determinado instante foram obtidas das equações citadas, derivando-as em relação ao tempo. Desse modo, as equações de taxa de infiltração instantânea (i) para cada modelo podem ser representadas conforme as equações (1) (2) e (3), respectivamente, para os modelos de Kostiakov-Lewis, Horton e Philip.

$$
\begin{aligned}
& i=\alpha \kappa t^{\alpha-1}+i_{f} \\
& i=i_{f}+\left(i_{o}-i_{f}\right) \exp ^{(-\beta t)} \\
& i=\beta+\frac{1}{2} \mathrm{kt}^{\left(\frac{-1}{2}\right)}
\end{aligned}
$$

em que,

$\mathrm{i}$ - taxa de infiltração em um determinado instante, $\mathrm{mm} \mathrm{h}^{-1}$;

$\mathrm{i}_{\mathrm{o}}$ - taxa de infiltração inicial, $\mathrm{mm} \mathrm{h}^{-1}$;

$\mathrm{i}_{\mathrm{f}}$ - taxa de infiltração final, $\mathrm{mm} \mathrm{h}^{-1}$;

$\mathrm{t}$ - tempo de infiltração, $\mathrm{h}, \mathrm{e}$

$\alpha, \beta$ e $\kappa$ - parâmetros determinados estatisticamente a partir de valores medidos de Infiltração (I)e t.

Os parâmetros $\alpha, \beta$ e $\kappa$ foram estimados por meio de regressão não linear, utilizando-se do método de Gauss-Newton (CHAPRA \& CANALE, 1998), utilizando-se dos valores das taxas de infiltração obtidos nos testes a campo. Foram utilizados, para analisar o grau de ajuste dos modelos, os seguintes índices estatísticos: coeficiente de massa residual (CMR), coeficiente de ajuste (CA) e eficiência (EF), representados pelas eqs.(4), (5) e (6):

$$
\begin{aligned}
& \mathrm{CMR}=\left(\sum_{\mathrm{i}=1}^{\mathrm{n}} \mathrm{O}_{\mathrm{i}}-\sum_{\mathrm{i}=1}^{\mathrm{n}} \mathrm{P}_{\mathrm{i}}\right) / \sum_{\mathrm{i}=1}^{\mathrm{n}} \mathrm{O}_{\mathrm{i}} \\
& \mathrm{CA}=\sum_{\mathrm{i}=1}^{\mathrm{n}}\left(\mathrm{O}_{\mathrm{i}}-\overline{\mathrm{O}}\right)^{2} / \sum_{\mathrm{i}=1}^{\mathrm{n}}\left(\mathrm{P}_{\mathrm{i}}-\overline{\mathrm{O}}\right)^{2} \\
& \mathrm{EF}=\left[\sum_{\mathrm{i}=1}^{\mathrm{n}}\left(\mathrm{O}_{1}-\overline{\mathrm{O}}\right)^{2} / \sum_{\mathrm{i}=1}^{\mathrm{n}}\left(\mathrm{O}_{1}-\overline{\mathrm{P}}\right)^{2}\right] / \sum_{\mathrm{i}=1}^{\mathrm{n}}\left(\mathrm{O}_{\mathrm{i}}-\overline{\mathrm{O}}\right)^{2}
\end{aligned}
$$

em que,

$\mathrm{O}_{\mathrm{i}}$ - valores observados;

$\mathrm{P}_{\mathrm{i}}$ - valores estimados;

n - número de observações, e

$\overline{\mathrm{O}}$ - média aritmética das observações. 


\section{RESULTADOS E DISCUSSÃO}

A umidade inicial do solo apresentou valores médios diferentes entre os tratamentos, sendo o maior valor de umidade inicial obtido no tratamento de colheita mecânica de cana crua, o que pode ter favorecido a menor taxa de infiltração inicial neste sistema (Tabela 2).

TABELA 2. Umidade do solo $\left(\%, \mathrm{~g} \mathrm{~g}^{-1}\right)$ e taxa de infiltração inicial e final $\left(\mathrm{mm} \mathrm{h}^{-1}\right) \mathrm{em}$ um Latossolo Vermelho distroférrico, nos respectivos sistemas de colheita da cana-deaçúcar. Soil moisture $\left(\%, \mathrm{~g} \mathrm{~g}^{-1}\right)$ and initial and final infiltration rate in the harvest systems of sugar cane in a Distroferric Red Latossol.

\begin{tabular}{lccc}
\hline & \multicolumn{3}{c}{ Tratamentos } \\
\hline Profundidade $(\mathrm{cm})$ & Manual Queimada & Mecânica Crua & Mecânica Queimada \\
\hline & \multicolumn{3}{c}{ Umidade inicial do solo } \\
\cline { 2 - 4 }-5 & 9,93 & 11,88 & 9,80 \\
$5-10$ & 9,72 & 14,40 & 10,06 \\
$10-20$ & 9,13 & 12,65 & 9,47 \\
Média & $9,59 \mathrm{~b}$ & $12,98 \mathrm{a}$ & $9,78 \mathrm{~b}$ \\
\hline & \multicolumn{4}{c}{ Taxa de infiltração $\left(\mathrm{mm}^{-1} \mathrm{~h}^{-1}\right)$} \\
Inicial & $57,21 \mathrm{a}$ & $48,24 \mathrm{~b}$ & $55,10 \mathrm{ab}$ \\
Final (TIB) & $45,67 \mathrm{a}$ & $12,67 \mathrm{~b}$ & $7,75 \mathrm{~b}$ \\
\hline
\end{tabular}

Médias seguidas de mesmas letras na linha não diferem estatisticamente, pelo teste de Tukey, a 5\% de probabilidade.

Os menores valores para taxa de infiltração básica (final) foram encontrados onde foi realizada a colheita mecânica de cana queimada. Tais resultados evidenciam a interferência de alguns atributos físicos do solo sobre a taxa de infiltração básica, especialmente a densidade e a macroporosidade (Tabela 1). A diminuição dos valores da taxa de infiltração básica pode ser explicada pela aplicação de maiores pressões na superfície do solo pelo tráfego de máquinas agrícolas nas áreas sob colheita mecanizada. BERGAMIN (2009) relata que o tráfego de máquinas induz ao aumento da compactação do solo e reduz sua macroporosidade. LUCA et al. (2008) verificaram que o uso de máquinas para a realização da colheita da cana-de-açúcar provocou aumento na densidade do solo nas camadas superficiais de um Argissolo Vermelho-Amarelo e um Neossolo Quartzarênico.

Por outro lado, no sistema de colheita manual de cana queimada, foram encontrados os maiores valores para a taxa de infiltração básica, o que está relacionado à menor compactação do solo, e consequentemente a melhores condições estruturais do solo, exercida por este tipo de colheita. ALMEIDA et al. (2009), avaliando o efeito de diferentes sistemas de colheita de cana-de-açúcar sobre algumas propriedades físicas do solo, observaram que o sistema de colheita manual com queima apresentou menor impacto nos atributos físicos do solo, com menor argila dispersa em água e maior índice de floculação, em comparação ao sistema de colheita de cana mecanizada sem queima da palha.

Quando comparados os dois sistemas onde foi empregada a colheita mecânica, verificou-se maior taxa de infiltração básica na colheita mecânica de cana crua, devendo-se tal fato à manutenção da cobertura vegetal sobre o solo. Segundo BARCELOS et al. (1999), os restos culturais sobre a superfície do solo interceptam e dissipam a energia cinética da chuva e do escoamento superficial, diminuindo a desagregação e o transporte de partículas, prevenindo a formação do selamento superficial, diminuindo a velocidade de escoamento, aumentando o tempo de contato da água com o solo, permitindo, assim, maior infiltração de água. Para BRANDÃO et al. (2007), a formação de crosta decorrente do impacto das gotas da chuva sobre o solo é um dos fatores que podem reduzir acentuadamente a infiltração e, consequentemente, aumentar $o$ escoamento superficial. 
Os maiores valores de taxa de infiltração foram obtidos na área onde se praticou a colheita manual de cana queimada, havendo redução de $72 \%$ na taxa de infiltração para colheita mecânica de cana crua e de $83 \%$ para colheita mecânica de cana queimada. Assim, pôde-se constatar que, apesar dos benefícios gerados pela manutenção da palhada sobre o solo no sistema de colheita mecânica de cana crua, existem influências negativas, exercidas pelo tráfego de máquinas sobre a taxa de infiltração de água no solo que precisam ser consideradas. SOUZA et al. (2005), avaliando o efeito de sistemas de colheita sobre os atributos físicos de um Latossolo Vermelho-Amarelo distrófico, também encontraram maiores valores para densidade e menores para macroporosidade no sistema de colheita mecânica de cana crua sem incorporação da palhada em relação à colheita manual de cana queimada. Nesse sentido, é interessante que se proponham manejos alternativos após a colheita mecanizada, para aliviar as condições de redução de macroporosidade e aumento de densidade do solo que afetam negativamente a infiltração de água no solo. Os parâmetros $\alpha, \beta$ e $\kappa$, ajustados para os modelos matemáticos estudados para explicar a infiltração de água no solo estudado, e os índices estatísticos a elas relacionados estão apresentados na Tabela 3.

TABELA 3. Parâmetros e índices estatísticos obtidos da estimativa da taxa de infiltração estável pelas equações de Horton, Kostiakow-Lewis e Philip. Parameters and statistical indexes obtained from the estimate infiltration rate by the equations of Horton Kostiakow-Lewis and Philip.

\begin{tabular}{|c|c|c|c|}
\hline \multirow{3}{*}{$\begin{array}{l}\text { Parâmetros e } \\
\text { Índices }\end{array}$} & \multicolumn{3}{|c|}{ Tratamentos } \\
\hline & Manual Queimada & Mecânica Crua & Mecânica Queimada \\
\hline & \multicolumn{3}{|c|}{ Modelo de Horton } \\
\hline$\beta$ & 0,0200 & 0,0900 & 0,1013 \\
\hline CMR & 0,0000 & 0,0600 & 0,0300 \\
\hline CA & 1,8100 & 1,7900 & 1,2900 \\
\hline \multirow[t]{2}{*}{$\mathrm{EF}$} & 0,0000 & 0,0000 & 0,0000 \\
\hline & \multicolumn{3}{|c|}{ Modelo de Kostiakov-Lewis } \\
\hline $\mathrm{a}$ & 0,6800 & 0,4807 & 0,3844 \\
\hline$k$ & 21,4466 & 86,8065 & 139,4586 \\
\hline CMR & $-0,0045$ & $-0,0343$ & $-0,0655$ \\
\hline $\mathrm{CA}$ & 3,0780 & 1,8311 & 1,5729 \\
\hline \multirow[t]{2}{*}{$\mathrm{EF}$} & 0,0002 & 0,0000 & 0,0000 \\
\hline & \multicolumn{3}{|c|}{ Modelo de Philip } \\
\hline$\beta$ & 47,5200 & 10,2400 & 2,5900 \\
\hline$k$ & 28,1000 & 91,1100 & 118,5300 \\
\hline CMR & 0,0000 & 0,0000 & 0,0000 \\
\hline $\mathrm{CA}$ & 2,8200 & 1,5600 & 1,3200 \\
\hline EF & 0,0000 & 0,0000 & 0,0000 \\
\hline
\end{tabular}

Observando o coeficiente de massa residual (CMR), verifica-se que, quando se utilizou a equação de Horton, a taxa de infiltração final pode ter sido subestimada, fato indicado pelos valores positivos do índice CMR. Para a equação de Kostiakov-Lewis, observaram-se valores superestimados da taxa de infiltração final em todos os tratamentos, o que foi indicado pelos valores negativos do índice CMR. Por outro lado, o melhor ajuste dos dados deu-se ao modelo da equação de Philip, uma vez que os valores de CMR determinados, quando se usou essa equação, foram iguais, a zero. O coeficiente de ajuste (CA) foi, em geral, melhor na equação de Horton, pois esse modelo foi o que apresentou valores mais próximos da unidade, exceto no sistema de colheita mecanizada de cana crua. Os valores do índice de eficiência (EF) foram semelhantes para os três modelos estudados, mostrando que não houve diferença entre as equações e entre os tratamentos. As curvas de taxa de infiltração obtidas em campo e as estimadas pelos modelos de Horton, Kostiakov-Lewis e Philip estão apresentados nas Figuras 1; 2 e 3. 


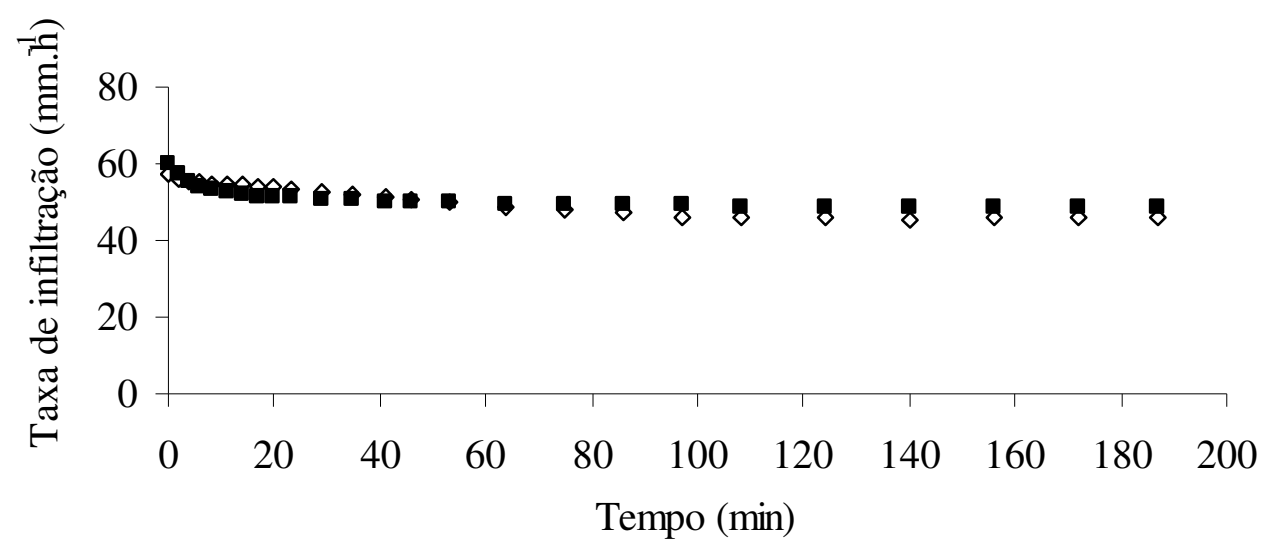

$\diamond$ dados observados - Kostiakov-Lewi $\left(\mathrm{R}^{2}=78,18\right)$

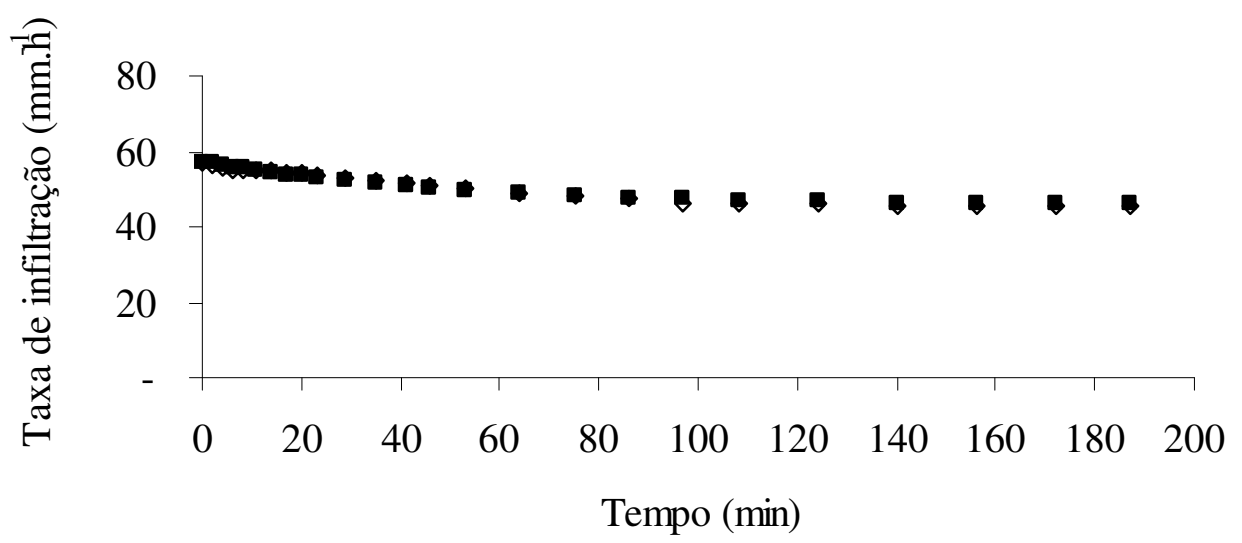

$\diamond$ Dados observados - Horton $\left(\mathrm{R}^{2}=98,75\right)$

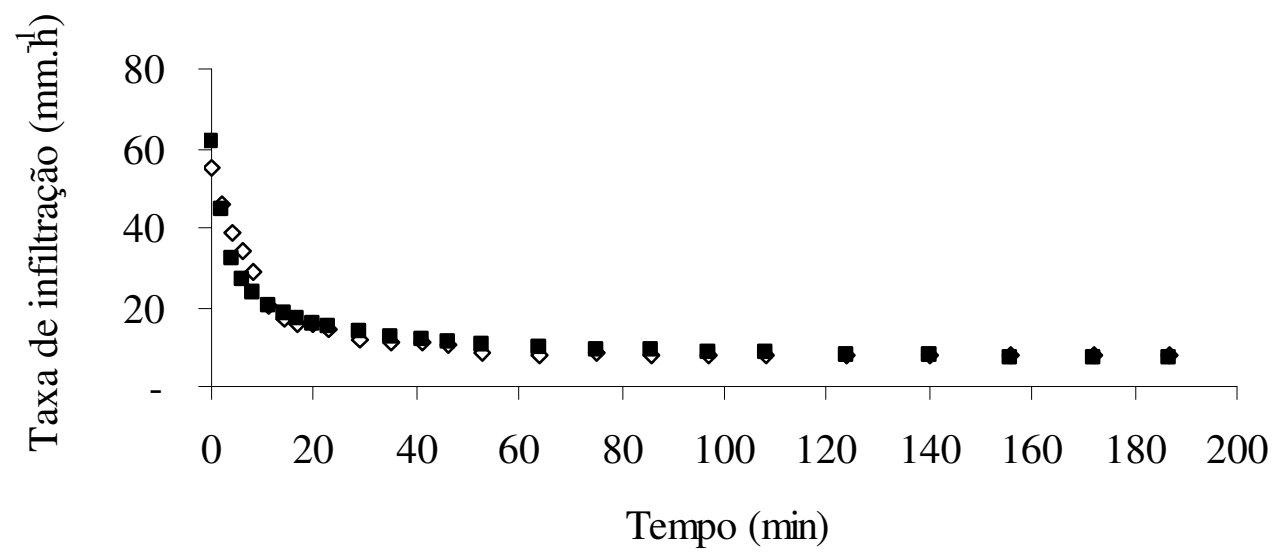

$\diamond$ Dados observados - Philip $\left(\mathrm{R}^{2}=61,38\right)$

FIGURA 1. Taxa de infiltração observada e estimada pelos modelos de Kostiakov-Lewis, Horton e Philip no sistema de colheita manual de cana queimada. Infiltration rate observed and estimated by the Kostiakov-Lewis, Horton and Philip models in the system of manual harvesting of burnt cane. 


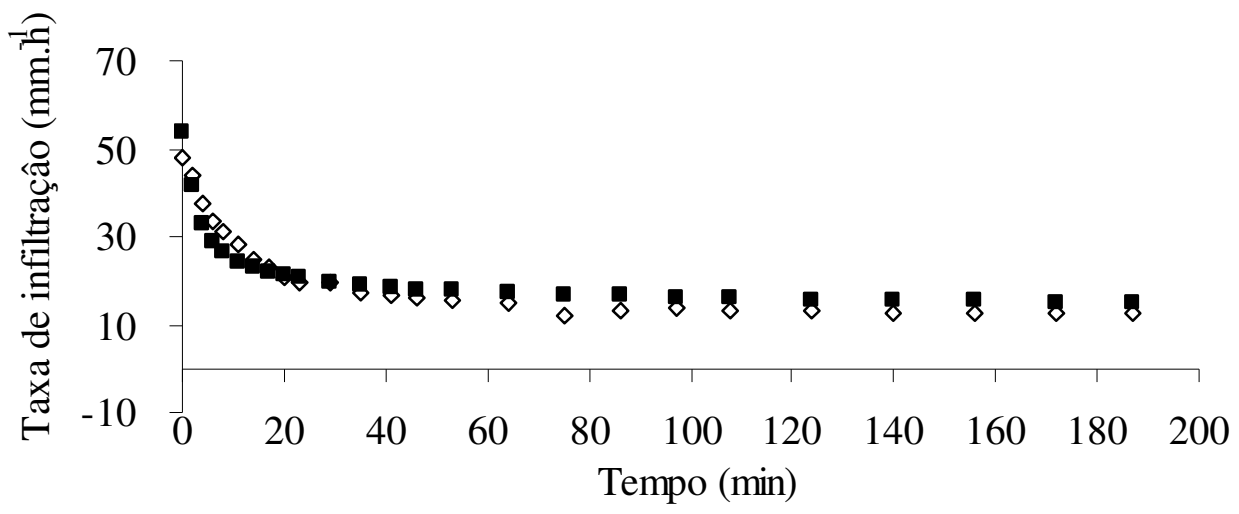

$\diamond$ dados observados - Kostiakov-Lewis $\left(\mathrm{R}^{2}=68,30\right)$

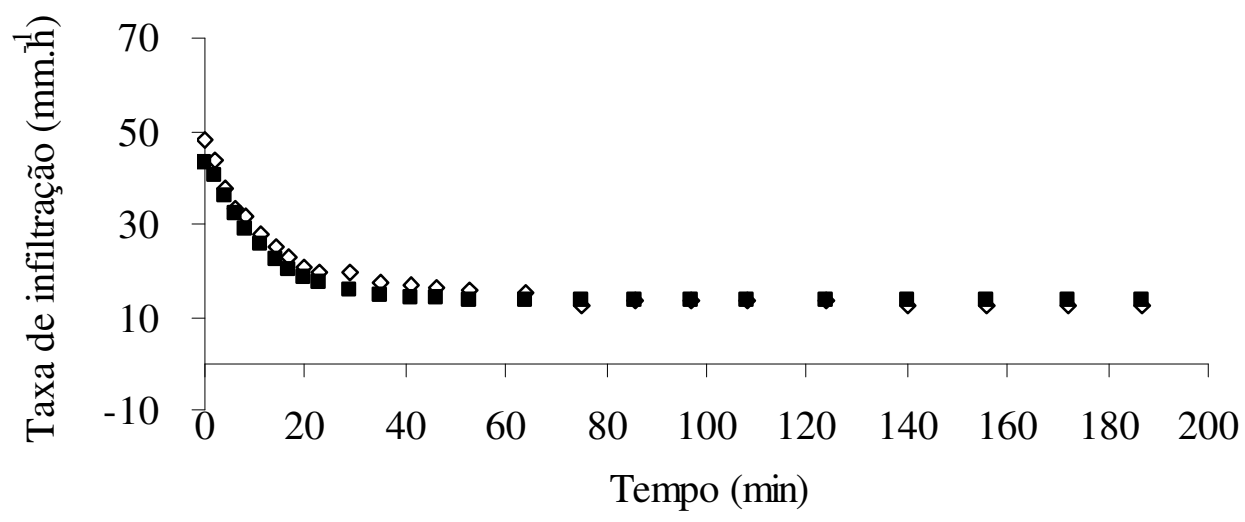

$\diamond$ Dados observados - Horton $\left(\mathrm{R}^{2}=72,15\right)$

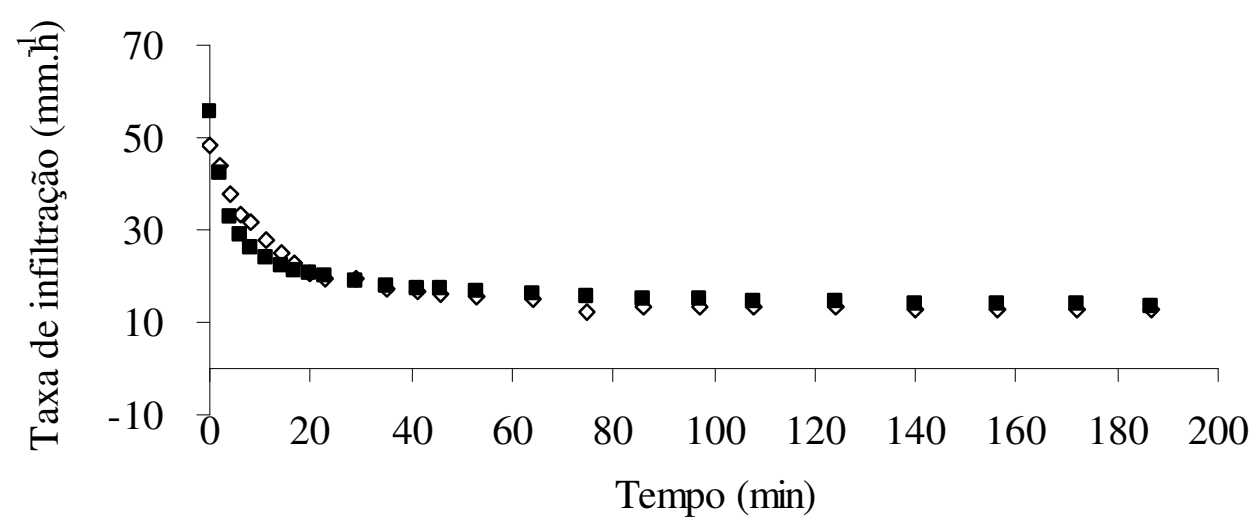

$\diamond$ Dados observados - Philip $\left(\mathrm{R}^{2}=61,38\right)$

FIGURA 2. Taxa de infiltração observada e estimada pelos modelos de Kostiakov-Lewis, Horton e Philip no sistema de colheita mecanizada de cana crua. Infiltration rate observed and estimated by the Kostiakov-Lewis, Horton and Philip model in the system of mechanized harvesting of raw cane. 


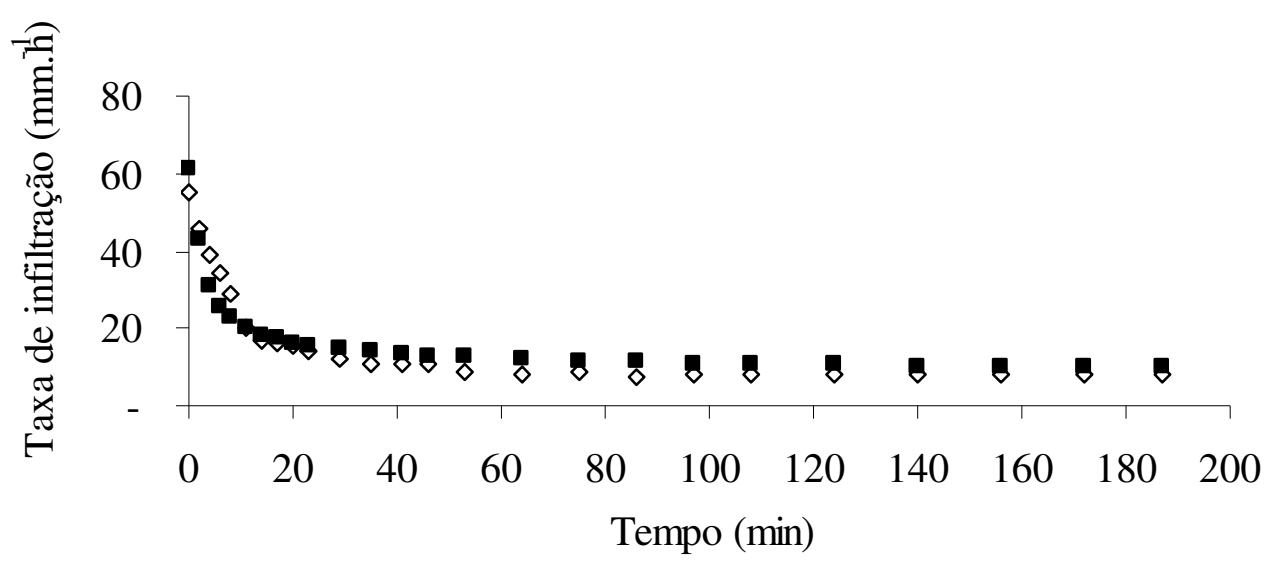

$\diamond$ dados observados - Kostiakov-Lewis $\left(\mathrm{R}^{2}=54,63\right)$

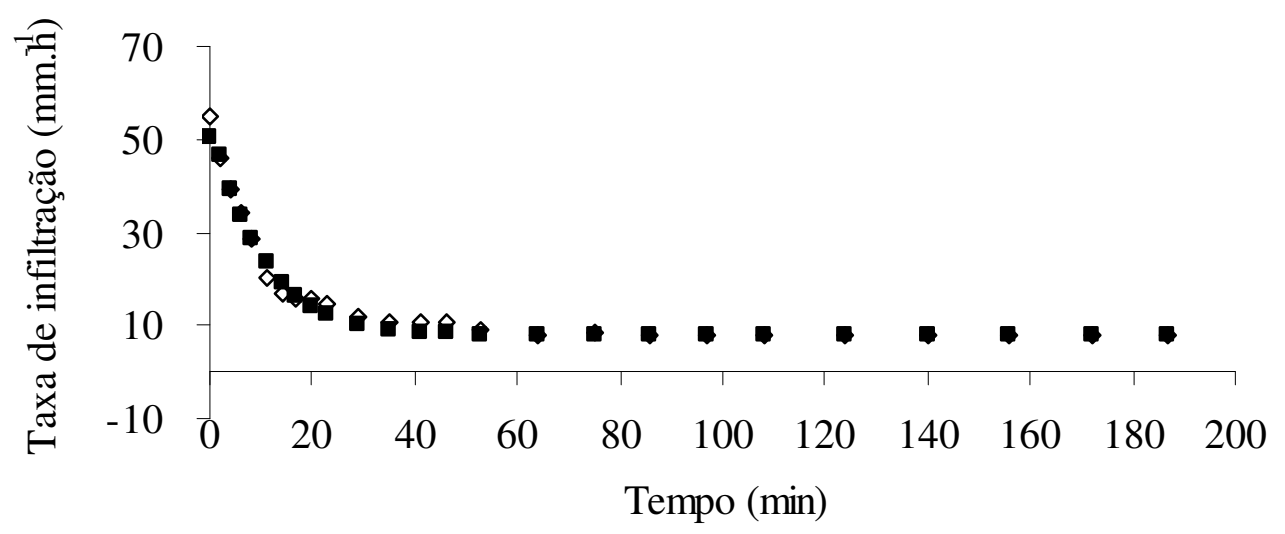

$\diamond$ Dados observados - Horton $(\mathrm{R} 2=68,99)$

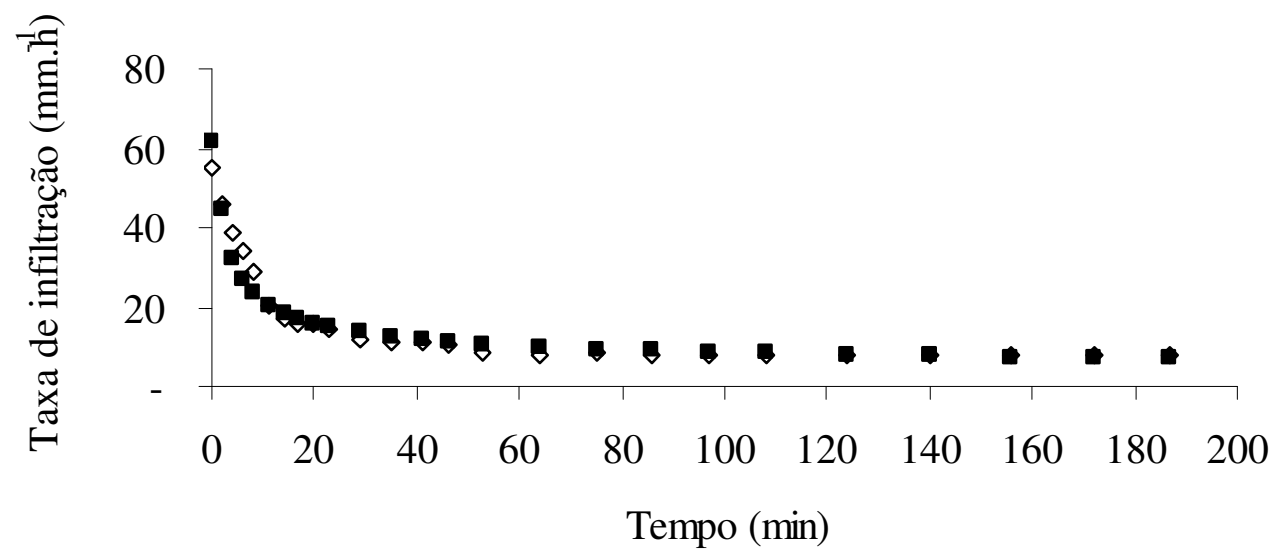

$\diamond$ Dados observados - Philip $(\mathrm{R} 2=61,38)$

FIGURA 3. Taxa de infiltração observada e estimada pelos modelos de Kostiakov-Lewis, Horton e Philip no sistema de colheita mecanizada de cana queimada. Infiltration rate observed and estimated by the Kostiakov-Lewis, Horton and Philip model in the system of mechanized harvesting of burnt cane. 
Pode-se afirmar que, de modo geral, a qualidade dos ajustes, medida pelo coeficiente de determinação $\left(\mathrm{R}^{2}\right)$, foi melhor para a equação de Horton em relação às equações de KostiakovLewis e de Philip, uma vez que, para a equação de Horton, os valores de $\mathrm{R}^{2}$ foram sempre superiores às demais equações. Trabalhando em dois sistemas de manejo do solo, ALVES SOBRINHO et al. (2003), por meio do uso do simulador de chuvas, avaliaram a adequação das equações de Horton e de Kostiakov-Lewis e concluíram que, para estimar a taxa de infiltração, nessas condições, a equação de Horton foi a mais adequada. Esses resultados sugerem que a equação de Horton, em se tratando de condições dinâmicas para a estimativa da taxa de infiltração de água, é a mais adequada.

\section{CONCLUSÕES}

O sistema de colheita mecanizada da cana-de-açúcar, quando comparado com a colheita manual de cana queimada, promoveu diminuição de $72 \%$ e $83 \%$ nos valores de infiltração de água no solo, respectivamente, para cana crua e queimada.

A equação de Horton foi a que melhor se ajustou aos dados de infiltração, confirmando essa equação como a mais indicada para a estimativa da taxa de infiltração no solo estudado.

\section{AGRADECIMENTO}

À Fundect-MS, pelo apoio financeiro ao projeto, e ao CNPq, pelas bolsas concedidas.

\section{REFERÊNCIAS}

ALMEIDA, C.X.; CENTURION, J.F.; JORGE, R.F.; ANDRIOLI, I.; VIDAL, A.A.; SERAFIM, R.S. Índice de floculação e agregação de um Latossolo Vermelho sob dois sistemas de colheita da cana-de-açúcar. Bioscience Journal, Uberlândia, v.25, n.4, p.123-129, 2009.

ALVES SOBRINHO, T.; FERREIRA, P.A.; PRUSKI, F.F. Desenvolvimento de um infiltrômetro de aspersão portátil. Revista Brasileira de Engenharia Agrícola e Ambiental, Campina Grande, v.6, n.2, p.337-344, 2002.

ALVES SOBRINHO, T.; VITORINO, A.C.T.; SOUZA, L.C.F.; GONÇALVES, M.C.;

CARVALHO, D.F. Infiltração de água no solo em sistemas de plantio direto e convencional.

Revista Brasileira de Engenharia Agrícola e Ambiental, Campina Grande, v.7, n.2, p.191-196, 2003.

BARCELOS, A.A.; CASSOL, E.A.; DENARDIN, J.E. Infiltração de água em um Latossolo Vermelho-Escuro sob condições de chuva intensa em diferentes sistemas de manejo. Revista Brasileira de Ciência do Solo, Viçosa-MG, v.23, N.1, p.35-43, 1999.

BEZERRA, S.A.; CANTALICE, J.R.B. Erosão entre sulcos em diferentes condições de cobertura do solo, sob cultivo da cana-de-açúcar. Revista Brasileira de Ciência do Solo, Viçosa-MG, 30, p.565-573, 2006.

BERGAMIN, A.C. Atributos físicos, sistema radicular e suas relações com a produtividade de milho em Latossolo Vermelho distroférico submetido à compactação induzida. $2009.70 \mathrm{f}$.

Dissertação (Mestrado em Agronomia) - Universidade Federal da Grande Dourados, Dourados, 2009.

BRANDÃO, V.S.; CECÍLIO, R.A.; PRUSKI, F.F.; SILVA, D.D. Infiltração da água no solo. 3. ed. Viçosa: UFV, 2006. 120 p.

BRANDÃO, V.S.; SILVA, D.D.; RUIZ, H.A.; PRUSKI, F.F.; SHAEFER, C.E.G.R.; MARTINEZ, M.A.; SILVA, E.O. Perda de solo e caracterização física e micromorfológica de crostas formadas em solos sob chuva simulada. Engenharia Agrícola, Jaboticabal, v.27, n.1, p.129-138, 2007. 
CEDDIA, M.B.; ANJOS, L.H.C.; LIMA, E.; RAVELLI NETO, A.; SILVA, L.A. Sistemas de colheita da cana-de-açúcar e alterações nas propriedades físicas de um solo podzólico amarelo no Estado do Espírito Santo. Pesquisa Agropecuária Brasileira, Brasília, v.34, n.8, p.1.467-1.473, 1999.

CHAPRA, S.C.; CANALE, R.P. Numerical methods for engineers: with programming and software applications. $3^{\text {rd }}$ ed. Boston: WCB/ MacGraw-Hill, 1998. 924 p.

EMBRAPA. EMPRESA BRASILEIRA DE PESQUISA AGROPECUÁRIA. Sistema brasileiro de classificação de solos. Brasília: Embrapa, 1999. 412 p.

IBGE. INSTITUTO BRASILEIRO DE GEOGRAFIA E ESTATÍSTICA. Ministério do

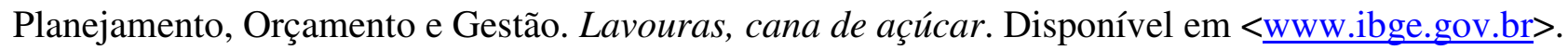
Acesso em: 01 jun. 2009.

LUCA, E.F.; FELLER, C.; CERRI, C.C.; BARTHÈS, B.; CHAPLOT, V.; CAMPOS, D.C.; MANECHINI, C. Avaliação de atributos físicos e estoques de carbono e nitrogênio em solos com queima e sem queima de canavial. Revista Brasileira de Ciência do Solo, Viçosa-MG, v.32, p.789800, 2008.

PAIXÃO, F.J.R.; ANDRADE, A.R.S.; AZEVEDO, C.A.V.; COSTA, T.L.; GUERRA, H.O.C. Ajuste da curva de infiltração por meio de diferentes modelos empíricos. Pesquisa aplicada \& Agrotecnologia, Guarapuava, v.2, n.1, p.108-112, 2009.

PANACHUKI, E.; ALVES SOBRINHO, T.; VITORINO, A.C.T.; CARVALHO, D.F.; URCHEI, M.A. Parâmetros físicos do solo e erosão hídrica sob chuva simulada em área de integração agricultura-pecuária. Revista Brasileira de Engenharia Agrícola e Ambiental, Campina Grande, v.10, n.2, p.261-268, 2006a.

PANACHUKI, E.; ALVES SOBRINHO, T.; VITORINO, A.C.T.; CARVALHO, D.F.; URCHEI, M.A. Avaliação da infiltração de água no solo, em sistemas de integração agricultura-pecuária, com uso de infiltrômetro de aspersão portátil. Acta Scientiarium Agronomy, Maringá, v.28, n.1, p.129137, 2006b.

SEVERIANO, E.C.; OLIVEIRA, G.C.; CURI, N.; DIAS JUNIOR, M. Potencial de uso e qualidade estrutural de dois solos cultivados com cana-de-açúcar em Goianésia (GO). Revista Brasileira de Ciência do Solo, Viçosa-MG, v.33, p.159-168, 2009.

SILVA, J.C.A.; ANDRADE, A.P.; SILVA, I.F. Avaliação da infiltração da água no solo como indicador de modificações edáficas em três sistemas de manejo. Agropecuária Técnica, João Pessoa, v.27, n.2, p.85-91, 2006.

SOUZA, Z.M. DE.; BEUTLER, A.N.; PRADO, R.M.; BENTO, M.J.C. Efeito de sistemas de colheita de cana-de-açúcar nos atributos físicos de um Latossolo Vermelho. Científica, Jaboticabal, v.34, n.1, p.31-38, 2006.

SOUZA, Z.M. DE; PRADO, R.M.; PAIXÃO, A.C.S.; CESARIN, L.G. Sistemas de colheita e manejo de palhada da cana-de-açúcar. Pesquisa Agropecuária Brasileira, Brasília, v.40, n.3, p.271278, 2005. 